\title{
OV And, a new field RRab Blazhko star?
}

\author{
K. Kolenberg ${ }^{1}$, E. Guggenberger ${ }^{1}$, P. Lenz ${ }^{1}$, P. Van Cauteren ${ }^{2}$, P. Lampens ${ }^{3}$, \\ P. Wils ${ }^{4}$ \\ ${ }^{1}$ Institut für Astronomie, Türkenschanzstr. 17, 1180 Vienna, Austria \\ ${ }^{2}$ Beersel Hills Observatory, Belgium \\ ${ }^{3}$ Koninklijke Sterrenwacht van België, Ringlaan 3, 1180 Ukkel, Belgium \\ ${ }^{4}$ Vereniging voor Sterrenkunde (VVS), Belgium
}

\begin{abstract}
Between 2004 November and 2005 January, we organized a small photometric observing campaign dedicated to the RRab star OV And. This star lies very close to other stars in the field. NSVS (Northern Sky Variability Survey) data suggested the presence of the Blazhko effect in this star. The new data reveal that the large discrepancies in pulsation amplitude of the star reported in the literature can be reproduced by applying different reduction methods. We show that only the data reduced with inclusion of the neighboring stars can be considered trustworthy. Nevertheless, we find some indications that the Blazhko effect may exist in this star.
\end{abstract}

\section{Introduction}

The most intriguing subclass of the astrophysically important RR Lyrae stars consists of stars showing the Blazhko effect, the phenomenon of amplitude or phase modulation. These stars have light curves that are modulated on timescales of typically tens to hundreds of days. Blazhko (1907) was the first to report this phenomenon in RW Dra. The estimated incidence rate of Blazhko variables among the galactic RRab stars (fundamental mode pulsators) is about 20-30 \% (Szeidl 1988; Moskalik \& Poretti 2002).

Though the Blazhko effect was discovered nearly a century ago, its physical origin still remains a mystery. Two groups of possible models are proposed, the magnetic models and the resonance models, but neither of them provides a sufficient explanation for the variety of behavior observed in Blazhko stars (see also Kolenberg 2004 for a concise general overview). 
The number of brighter Blazhko stars in the field is limited, and most of them have been known for a long time. For some, extensive photometric studies have been published, often using data sets gathered over several decades (e.g., Borkowski 1980, Kovacs 1995, Szeidl \& Kollath 2000, Smith et al. 2003, Jurcsik et al. 2005). Long-term photometric surveys, often serving other aims than studying only stellar variability (e.g., microlensing surveys like MACHO - Alcock et al. 2003, and OGLE - Moskalik \& Poretti 2002), have entailed the detection of a multitude of new variable stars, among which also RR Lyrae Blazhko stars. As a by-product of the Robotic Optical Transient Search Experiment (ROTSE), the Northern Sky Variability Survey (NSVS) was established, offering a temporal record of the sky over the optical magnitude range from 8 to 15.5 . This offers data for detecting new RR Lyrae Blazhko stars in the field. One of such suspected Blazhko candidates is OV And.

\section{The target star OV And}

In the sky plane the RR Lyrae star OV And (NSV 134, RA2000: 00:20:44.42, Dec2000: 40:49:41.6, V=10.4-11.0 according to GCVS 2003) lies close to a few other stars (Rössiger 1987), which makes it difficult to resolve with the classical photometric technique involving aperture photometry. Together with its 'companion' of almost equal brightness (at an angular separation of 7.5 arcsec) the star was previously denoted as BD+40 $0060=$ NSV 134 (Nikulina 1959) and was supposed to be a rapid irregular variable in the New Catalogue of Suspected Variable Stars (Kukarkin \& Kholopov 1982). Photometric data from 1985-1986 revealed a light curve amplitude of about $0.5 \mathrm{mag}$, and led to the conclusion that one of the stars must be an RR Lyrae star of type 'ab', meaning that it pulsates in the fundamental radial mode (Rössiger 1987).

From CCD images obtained by Prosser (1988) it was discovered that in fact there are three other fainter stars located near the variable and its bright 'companion'. High-dispersion spectra obtained by the same author revealed a large difference in radial velocities between OV And (about $-194 \mathrm{~km} / \mathrm{s}$ ) and its bright 'companion' (-62 km/sec), indicating that the two brighter stars do not form a physical pair. In his photometric data, which allowed resolution of OV And and its 'companion', no variability was detected in the 'companion' nor in any of the other neighboring stars. Prosser's differential observations (featuring 'variable minus 'companion' magnitude') point out an amplitude of 0.980 mag ( $\sigma=0.004 \mathrm{mag}$ ) in the star's light variation, essentially twice the amplitude found by Rossiger. According to Prosser this "could mean that the adopted comparison star, BD+40 0056, may have some variation in brightness as well". Neither author mentioned a Blazhko effect. 


\subsection{NSVS data of OV And}

The NSVS data of OV And were gathered by ROTSE-I in 1999-2000, and cover a total time span of 254.8 days.

A frequency analysis of the 155 NSVS measurements with Period04 (see Lenz \& Breger 2005, this volume) yields the main frequency $f$ to the expected accuracy. We adopt the period $P=0.470581 \mathrm{~d}$ (frequency $f=2.12503$ $\mathrm{c} / \mathrm{d}$ ) listed in the GEOS RR Lyrae database and determined from previous measurements.

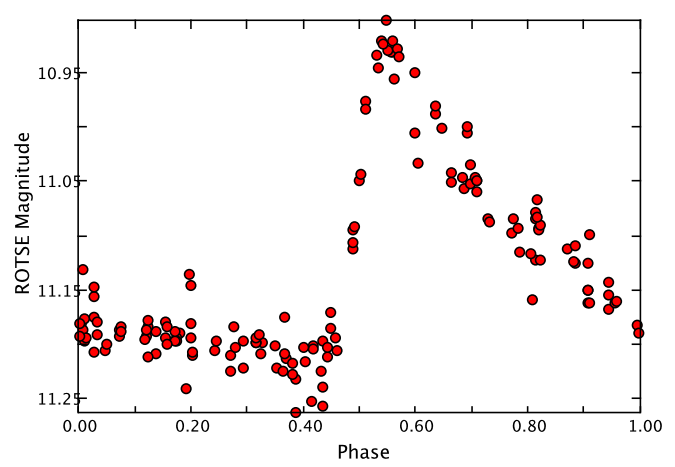

Figure 1: NSVS data phased with the main frequency $f=2.12504 \mathrm{c} / \mathrm{d}$. For visibility we chose phase 0.5 to be in the middle of the rising branch. Is the large scatter partly due to a Blazhko effect?

In all our frequency analyses we adopt a $4 \sigma$ significance criterion, $\sigma$ being the mean noise level in the Fourier periodogram at the relevant frequency (Breger et al 1993). After prewhitening with the known main frequency and its significant first 5 harmonics, a new frequency peak is found close to the main frequency, however only at a level of $3.5 \sigma$. Such closely-spaced frequency peaks are a typical feature of Blazhko stars. However, due to its low signal-to-noise ratio, we are not (yet) convinced that this peak is related to a real frequency.

As the pixel size of the CCD used for the NSVS corresponds to about 14 arcsec on the sky, the ROTSE-I cameras will always have measured the combined magnitude of all five stars in the small group, resulting in a smaller amplitude. Hence, the Blazhko effect should become harder to detect. The light curve folded with the main period (Figure 1) shows a large scatter. The average point to point photometric scatter of the NSVS data is reported to be \pm 0.02 mag for bright, unsaturated stars (Woźniak et al. 2004). 
Table 1: Log of the photometric observations of OV And. BHO: Beersel Hills Observatory - vlt: Vienna Little Telescope.

\begin{tabular}{llll}
\hline Observatory & Detector & Reduction Software & Observers \\
\hline BHO & SBIG ST10XME & Mira AP (1) & PVC, PLa \\
vlt & Apogee AP9E & MaxIm DL (2), IRAF (3) & KK, EG, PLe \\
\hline
\end{tabular}

(1) Produced by Axiom Research Inc. (2) Produced by Diffraction Limited.

(3) Image Reduction and Analysis Facility,

written and supported at the National Optical Astronomy Observatories (NOAO).

\begin{tabular}{lccccc}
\hline $\begin{array}{l}\text { Date/HJD } \\
=2450000\end{array}$ & Telescope & Observatory & Filters & \multicolumn{2}{c}{ Amount of data } \\
Data & Hours \\
\hline $19.11 .04 / 3329$ & $25-\mathrm{cm}$ & $\mathrm{BHO}$ & $V$ & 118 & 4.089 \\
$24.11 .04 / 3334$ & $25-\mathrm{cm}$ & $\mathrm{BHO}$ & $V$ & 32 & 1.078 \\
$24.11 .04 / 3334$ & $80-\mathrm{cm}$ & $\mathrm{vlt}$ & $V$ & 75 & 2.539 \\
$25.11 .04 / 3335$ & $80-\mathrm{cm}$ & $\mathrm{vlt}$ & $B, V$ & 77,80 & $7.576,7.649$ \\
$03.12 .04 / 3343$ & $40-\mathrm{cm}$ & $\mathrm{BHO}$ & $V$ & 108 & 3.156 \\
$20.12 .04 / 3360$ & $80-\mathrm{cm}$ & vlt & $B, V$ & 91,91 & $4.937,4.937$ \\
$21.12 .04 / 3361$ & $80-\mathrm{cm}$ & vlt & $B$ & 150 & 3.901 \\
$11.01 .05 / 3382$ & $80-\mathrm{cm}$ & vlt & $B, V$ & 38,35 & $3.788,3.829$ \\
$17.01 .05 / 3388$ & $80-\mathrm{cm}$ & vlt & $B, V$ & 18,21 & $2.627,2.586$ \\
\hline
\end{tabular}

Total time span: $B 53.1 \mathrm{~d}, V 59.1 \mathrm{~d}$.

We decided to start a photometric monitoring of OV And in order to check whether a Blazhko effect is indeed present.

\section{Photometry of OV And}

\subsection{Observations}

Photometric CCD observations were gathered from two different sites: the 80-cm telescope at the Vienna Observatory (Austria) yielded Johnson $B$ and $V$ data, and the $25-\mathrm{cm}$ and $40-\mathrm{cm}$ telescopes at Beersel Hills Observatory (Belgium) provided data in the filter $V$ (following Bessell's specifications, Bessell 1995) only.

A log of the observations is given in Table 1. We used GSC 27871798 as the comparison star and GSC 27871777 as the check star. The errors on individual data points varied between $0.010 \mathrm{mag}$ and $0.030 \mathrm{mag}$ as obtained 
Table 2: Observed times of maximum (in Johnson $B$ and $V$ filters), and corresponding O-C values for the elements $T_{0}=H J D 24539026.478$ and $P=0.470581 \mathrm{~d}$ from the GEOS RR Lyrae database. Errors in HJD(Max) are of order $0.001 \mathrm{~d}$.

\begin{tabular}{cc}
\hline HJD $($ Max $)$ & O-C $( \pm 0.0014 \mathrm{~d})$ \\
\hline 2453329.306 & -0.0105 \\
2453334.476 & -0.0169 \\
2453335.418 & -0.0161 \\
2453360.358 & -0.0169 \\
2453361.300 & -0.0160 \\
\hline
\end{tabular}

from the rms error on the differential magnitudes of the comparison star and the check star.

\subsection{Analysis of the data}

On the CCD frames obtained with the $25-\mathrm{cm}$ and $40-\mathrm{cm}$ telescopes it was impossible to resolve OV And and its 'companion', and hence the reduced data represent the combined light. For the CCD frames obtained in Vienna we carried out the reductions twice: once with an aperture radius of 7 pixels (i.e., about 0.5-0.8*FWM depending on seeing conditions) which allowed to (barely) separate both stars and once with an aperture radius of 19 pixels (about 3.5*FWHM) including both stars.

Rössiger (1987) found the following elements from his observations:

$$
\operatorname{HJD}(\operatorname{Max})=2446764.241( \pm 0.001)+0.470568( \pm 0.000002) \mathrm{E}(\mathrm{d}),
$$

confirmed by Prosser (1988) based on data taken one year later. Adopting Rössiger's period, our observed times of maximum do no longer correspond with his time $T_{0}$ of maximum light. Adopting the period $P=0.470581 \mathrm{~d}$ listed in the GEOS database, we still obtain a systematic shift in the $(\mathrm{O}-\mathrm{C})$ values (Table 2). As an updated ephemeris we propose:

$$
\operatorname{HJD}(\operatorname{Max})=2453335.418( \pm 0.001)+0.470580( \pm 0.000001) \mathrm{E}(\mathrm{d}) \text {. }
$$

\subsubsection{How efficiently can the stars be resolved in the reduction?}

For the night with the best seeing (JD 245 3361), we were able to separate OV And from its 'companions', as illustrated in Fig. 3 displaying the 'OV And group' on representative frames for two different nights. The bright 'companion' star indeed proved to be constant, as was already reported by Prosser (1988). 

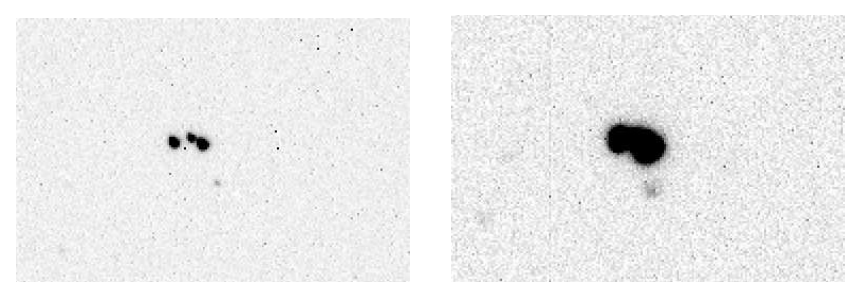

Figure 2: OV And (star to the E) and its immediate neighborhood on representative frames obtained at the 80-cm telescope in Vienna for HJD 2453361 (left) and HJD 2453335 (right). This figure demonstrates the effect of variable seeing. (North is up, East is left.)

For all other nights, the differential data of the 'companion' star showed variations in brightness. However, we found evidence that these variations are linked to those seen in the OV And data and therefore must be caused by some contribution of the light of OV And itself. In the same way, the variable contribution of the 'companion(s)' in the 'separated' OV And data (due to variable seeing conditions) may not be neglected, as already indicated by the larger scatter in the data, and hence these reduced data have to be interpreted with caution!

The peak-to-peak $B$ amplitude for the night of JD 2453361 amounts to $1.65 \pm 0.03 \mathrm{mag}$. As we only obtained $B$ data during this night, we can make no statements on the $V$ magnitude of OV And (without additional light).

\subsubsection{Is OV And a Blazhko star?}

Blazhko stars are typified by the occurrence of secondary frequencies close to the main pulsation frequency and its harmonics. These frequencies are thought to be related to nonradial modes in the mainly radially pulsating RR Lyrae star, modes that, according to the currently plausible models, would cause the observed amplitude and/or phase modulation.

A period analysis of the OV And $B$ and $V$ data, respectively with its 'companions' and tentatively separated, and using Period04, always revealed the main frequency $f$ and its harmonics, up to high (8th) order. However, we did not find any evidence of a secondary frequency close to the main pulsation frequency above the $4 \sigma$ significance level.

The main feature of the Blazhko effect is the variation of the light curve shape and amplitude. Table 3(a) displays the total amplitude of the lightcurve in different nights. From these data we cannot unambiguously conclude on the presence of a Blazhko effect in OV And yet; at the level of $2 \sigma$ the amplitudes 
Table 3: Variation of the (peak-to-peak) light curve amplitude in the Johnson $B$ and $V$ filter. The data in the upper panel are for OV And with its 'companions'; the lower panel data for the reduced data in which we attempted to separate the light variation of OV And. Note that the upper panel reproduces Rössiger's (1987) values quite well, whereas the lower panel agrees better with Prosser's (1988) values. However, we call for caution in interpreting the amplitudes in the lower panel!

\begin{tabular}{l|cccc}
\hline (a) & \multicolumn{5}{|c}{ OV And and 'companions' } \\
Date & $A_{V}(\mathrm{mag})$ & $\Delta A_{V}$ & $A_{B}(\mathrm{mag})$ & $\Delta A_{B}$ \\
\hline 19.11 .04 & 0.476 & 0.015 & & \\
24.11 .04 & 0.463 & 0.015 & & \\
25.11 .04 & 0.458 & 0.015 & 0.865 & 0.015 \\
20.12 .04 & 0.460 & 0.015 & 0.894 & 0.015 \\
21.12 .04 & \multicolumn{5}{|c}{0.893} & 0.015 \\
\hline \hline (b) & \multicolumn{5}{c}{ OV And 'separated' } \\
Date & $A_{V}$ (mag) & $\Delta A_{V}$ & $A_{B}(\mathrm{mag})$ & $\Delta A_{B}$ \\
\hline 24.11 .04 & 1.015 & 0.028 \\
25.11 .04 & 1.053 & 0.057 & 1.505 & 0.028 \\
20.12 .04 & 1.179 & 0.057 & 1.596 & 0.028 \\
21.12 .04 & 1.179 & 0.028 & 1.655 & 0.028 \\
\hline
\end{tabular}

are stable. Though there may be an indication for a variation of the total amplitude as suggested by the data in Table3(b), we call for much caution at this stage. We indeed cannot trust the values from Table 3(b) as it is very difficult to obtain "clean" measurements of OV And without influence of the 'companion"s light (and other neighboring stars)!

\section{Conclusions}

- Like previous authors who used small telescopes, we experienced difficulties separating OV And from its neighbors on the sky. Reduction of data of OV And should include all neighboring stars in order to be trustworthy.

- The smaller amplitude reported by Rössiger (1987), about half of the amplitude detected by Prosser (1988), can be explained by the fact that Rössiger included the bright 'companion' in his analysis, rather than due to the comparison star's behavior, as Prosser (1988) assumed. Additional star light will reduce the total amplitude of the light curve, and also influences its shape. The NSVS data show an even smaller amplitude. The main reason for this, besides the inclusion of yet another star in the reduction, is that the ROTSE camera (unfiltered CCD) is more sensitive 


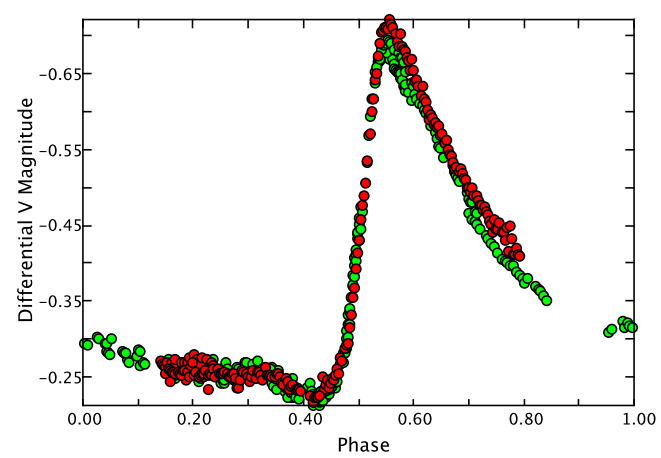

Figure 3: All our $V$ data phased with the main frequency. OV And was reduced with its 'companions' included in the aperture. The amplitudes of the lightcurve are listed in Table 3(a) (see columns for $V$ ). The data suggest a small change in the light curve shape and amplitude, but its significance needs to be proven.

in the red, the wavelength region where RR Lyrae stars emit less light than in $V$.

- For the 'isolated' OV And, we detected a light curve amplitude of $1.6 \pm 0.1$ mag in the $B$ filter. The light curve in the $V$ filter is estimated to be $1.1 \pm 0.1 \mathrm{mag}$.

- The present data don't confirm the additional frequency suggested by the NSVS data, but since we only observed 4 maxima we cannot disprove its reality yet. Moreover, our observed light curves suggest that a Blazhko effect may be present. We plan a follow-up action on OV And for next autumn.

\section{The Blazhko Project}

These observations were carried out in the framework of the Blazhko Project, an international collaboration, set up to join efforts in obtaining a better understanding of the Blazhko phenomenon in RR Lyrae stars. The project was founded in Vienna and started its activities in the autumn of 2003. The starting point for improving the modeling is an extensive data set, consisting of spectroscopy and photometry, of a limited sample of field RR Lyrae Blazhko stars, and also some non-Blazhko stars for comparison. Besides the larger campaigns, 
some small photometric campaigns - like this one - are organized to fine-tune our knowledge of the frequency behavior of field Blazhko stars.

All interested colleagues - professionals and amateurs - are warmly invited to join in the observational campaigns of the Blazhko Project. For more information we refer to the website dedicated to the Blazhko Project

(http://www.astro.univie.ac.at/ blazhko/)

or directly contact kolenberg@astro.univie.ac.at.

Acknowledgments. The Blazhko Project, KK and EG are supported by the Austrian Fonds zur Förderung der wissenschaftlichen Forschung, project number P17097-N02. KK thanks Jacqueline Vandenbroere and Anton Paschke for information on the data reported on the GEOS and BAV websites.

The data were partly gathered in the framework of the Observatoriumspraktikum, during which students at the Institute for Astronomy are taught to use the telescope and CCD camera (see http://www.deltascuti.net/obsprak/ and http://www.astro.univie.ac.at/ blazhko/Winter.html). We thank all the students who participated in the observations of OV And: Paul Beck, Daniel Blaschke, Johannes Puschnig, Markus Hareter, Eveline Glassner, Pia Hecht, Elisabeth Füllenhals, Sophie Aspöck, Vera Steinecker, Gerald Jungwirth, Hanns Petsch, Jenny Feige, Adrian Partl, Mathias Jäger, Petra Zippe, Marius Halosar, Florian Herzele. Part of the data was acquired with equipment purchased thanks to a research fund financed by the Belgian National Lottery (1999).

\section{References}

Alcock, C., Allsman, R.A., Becker, A., et al., ApJ 598, 597

Bessell, M.S., 1995, CCD Astronomy 2, No. 4, 20

Blazhko, S., 1907, Astron. Nachr. 175, 325

Borkowski, K.J., 1980, Acta Astron. 30, 393

Breger, M., Stich, J., Garrido, R., et al., 1993, A\&A 271, 482

GEOS RR Lyrae database: http://webast.ast.obs-mip.fr/people/leborgne/dbRR/ Kolenberg, K., 2004, CoAst 145, 16

Kukarkin, B.V., Kholopov, P.N., 1982, New Catalogue of Suspected Variable Stars Jurcsik, J., Sódor, Á., V'aradi, M., et al., 2005, A\&A 430, 1049

Kovacs, G., 1995, A\&A 295, 693

Lenz, P., \& Breger, M., 2005, CoAst 146, 53

Moskalik, P., \& Poretti, E., 2002, A\&A398, 213

Nikulina, T.G., 1959, Astron. Tsirk., 207, 14

NSVS, Northern Sky Variability Survey, http://skydot.lanl.gov/nsvs/nsvs.php

Prosser, C.F., 1988, Inform. Bull. Var. Stars, 3130, 1

Rossiger, S., 1987, Inform. Bull. Var. Stars, 2977, 1

ROTSE, Robotic Optical Transient Search Experiment,

http://rotse1.physics.lsa.umich.edu/ 
Szeidl, B., 1988, in 'Multimodal Stellar Pulsations', Kultura, eds. Kovacs, G., Szabados, L., Szeidl, B., 45

Szeidl, B., Kollath, Z., 2000, ASP Conf. Ser. 203, 281

Smith, H.A., Church, J.A., Fournier, J., et al., 2003, PASP 115, 43

Woźniak, P. R., Vestrand, W. T., Akerlof, C. W., et al., 2004, The Astronomical Journal, Vol. 127, 2436 